\title{
The VTRx+, an Optical Link Module for Data Transmission at HL-LHC
}

\author{
Jan Troska*, Alexander Brandon-Bravo, Stéphane Detraz, Andrea Kraxner, Lauri \\ Olanterä, Carmelo Scarcella, Christophe Sigaud, Csaba Soòs, and François Vasey \\ CERN \\ Route de Meyrin, $\mathrm{CH}-1211$ Geneva 23 \\ E-mail: jan.troskaecern.ch
}

Optical data transmission will remain a key enabling technology for the upgrading detectors at HL-LHC. In particular the inner tracking detectors will require low-mass, radiation tolerant optical transmit and receive modules for tight integration in the detector front-ends. We describe the development of such a module, giving details of the design, functional and environmental performance, as well as showing the feasibility of achieving small size, low-mass, and low-power operation.

Topical Workshop on Electronics for Particle Physics

11 - 14 September 2017

Santa Cruz, California

\footnotetext{
* Speaker.
} 


\section{Introduction}

During the phase II upgrades of the ATLAS and CMS experiments at the Large Hadron Collider (LHC) several detectors will be replaced to improve their physics performance. In particular, these upgrades aim to replace the innermost detectors that are exposed to the harshest radiation environments. To cope with the increasing data volume and higher trigger rate, high-speed optical links will again be deployed in large quantities as part of the upgrade programme. The tight space constraints and the high channel count of the on-detector electronics will require the development of a low-profile (20 mm x $10 \mathrm{~mm} \times 2.5 \mathrm{~mm}$ target), multi-channel front-end component. During their expected lifetime these components will have to withstand the on-detector radiation levels (1MGy total dose, $1 \times 10^{15} \mathrm{n} / \mathrm{cm}^{2}$ and $1 \times 10^{15} \mathrm{~h} / \mathrm{cm}^{2}$ total fluence) and they have to operate over a wide temperature range $\left(-35^{\circ} \mathrm{C}\right.$ to $\left.65^{\circ} \mathrm{C}\right)$. The Versatile Link PLUS (VL+) project is developing a radiation-tolerant optical link system shown schematically in figure 1 that fulfils these requirements. The front-end module (VTRx+) will be based on radiation-hard laser diode driver (LDD) and transimpedance amplifier (TIA) ASICs, and commercial VCSEL and PIN photodiode (PD) components. A set of passive components (optical fibre and connectors) will connect the VTRx+ to the off-detector electronics where Commercial Off-The-Shelf (COTS) optical transmitter and receivers will provide the interface to FPGAs. The VL+ is designed to operate together with the lpGBT Serializer/Deserializer (SerDes) [1], although other SerDes types can be supported. The target operating data-rates for the $\mathrm{VL}+$ are set by the lpGBT: $2.56 \mathrm{~Gb} / \mathrm{s}$ in the downstream towards the detector; and 5.12 or $10.24 \mathrm{~Gb} / \mathrm{s}$ in the upstream away from the detector.
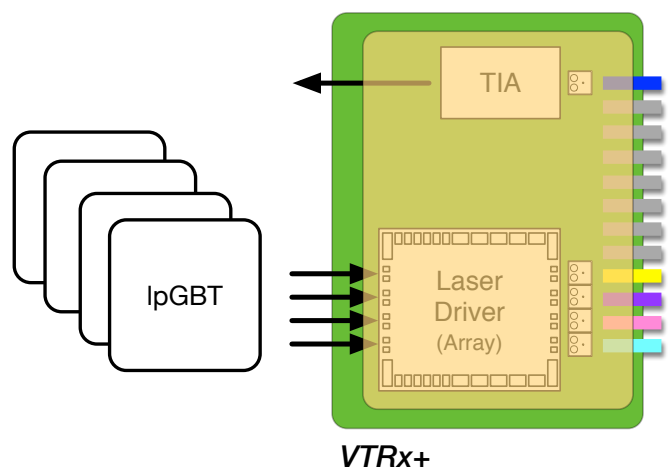

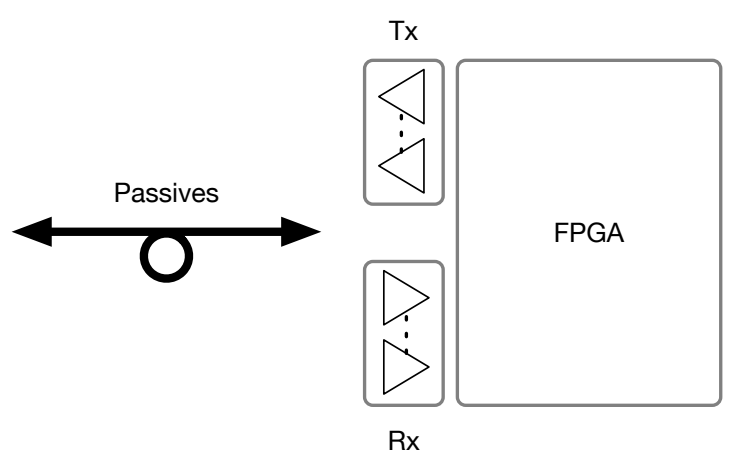

Figure 1: Overview of the Versatile Link + system showing the components of the VTRx+.

The VL+ project is a collaborative venture with partners from CERN (CH), FNAL (US), Oxford University (GB), Southern Methodist University (US), and Academia Sinica (TW). Within the $\mathrm{VL}+$ project CERN is responsible for the delivery of the VTRx+ module and its optoelectronic components. This paper describes the development of the VTRx+ module. The details of the full environmental and functional validation of candidate optoelectronic components that will be used on the VTRx+ module will be the subject of a future publication.

\section{VTRx+ module}

The VTRx+ module will be a small form factor module that will meet the dimensional and 
environmental constraints given by the various upgrading sub-detectors of ATLAS and CMS at HLLHC. There will be three operational variants of the module that differ in their configuration of the number of Transmitter (Tx) and Receiver (Rx) channels that will be used in operation. Following a survey of potential users, out of a total of approximately $50000 \mathrm{VTRx}+$ modules, $60 \%$ require a 1 Tx and $1 \mathrm{Rx}$ configuration, $12 \%$ a $4 \mathrm{Tx}$ and $1 \mathrm{Rx}$ configuration, and $28 \%$ a $4 \mathrm{Tx}$ only configuration. In order to simplify the module design we have chosen to concentrate on a basic module that could meet the 4 Tx and 1 Rx configuration since the other two can be achieved by either simply disabling channels and/or perhaps removing the PD and TIA. A critical building block of any optical transceiver module is the optical coupling unit that turns the vertically-emitted light from the VCSEL through $90^{\circ}$ and focuses it into the optical fibre (and performs the analogous function for the PD). Commercially-available optical modules on which the VTRx+ could be based are typically 12-channel Tx or Rx, or 4 Tx and 4 Rx modules, thus containing optical coupling blocks that enable the VTRx+ configurations listed above. Figure 2 shows the three configurations of VTRx+ based upon such an optical coupling block and the use of the LDQ10 laser driver [2] and the GBTIA [3]. Although the GBTIA was designed for the Versatile Link project $[4,5]$ for operation at $4.8 \mathrm{~Gb} / \mathrm{s}$ when packaged with a PD directly into a TO-can based ROSA, it is possible to use it in a single-channel configuration for the VTRx+.
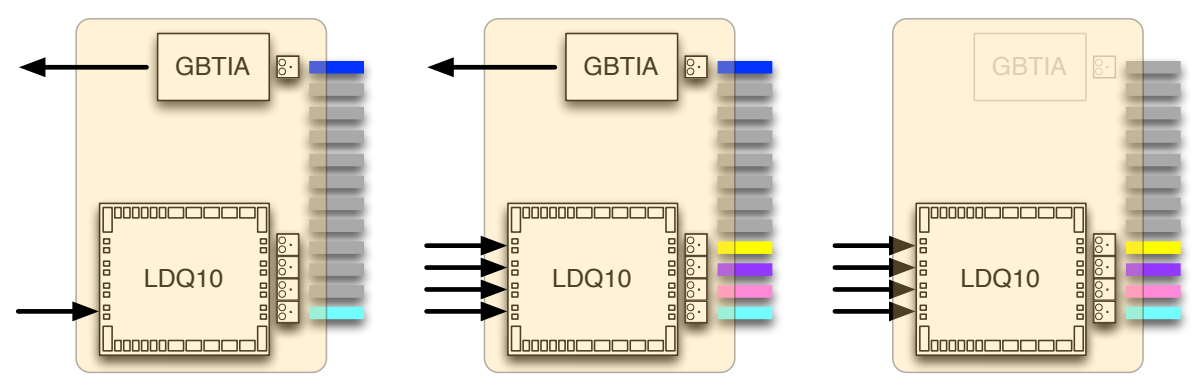

Figure 2: Configurations of the VTRx+: (left) single Tx/Rx; (centre) quad Tx \& single Rx; and (right) quad Tx only. Grey fibres are unused and the semi-opaque box represents the optical coupling block.

One of the critical parameters of the VTRx+ for a number of the end users is its height. The height is also one of the more challenging dimensions to reduce when employing an optical coupling block that must turn the light through $90^{\circ}$ to couple to the output optical fibre. Because of the challenging nature of reducing the height, two variants of module height are currently being considered: $2.5 \mathrm{~mm}$ and $4.5 \mathrm{~mm}$. The larger height is being maintained in an attempt to keep the overall cost per module down in production volumes. Should it turn out that the less tall variant can be produced cost-effectively then only that one could be taken into final production.

\section{VTRx+ module prototyping}

Two parallel development paths have been followed in order to develop VTRx+ prototypes. The first path combines commercially available VCSELs, PDs, and optical coupling blocks with a PCB designed in-house to host the LDQ10 and GBTIA. The second path builds upon the expertise available in industry and aims to work with industrial partners to integrate the LDQ10 and GBTIA 
into their pre-existing transceiver modules. Both paths have yielded functional modules that are described in more detail below.

\subsection{In-house VTRx+module}

Three variants of in-house design have been successfully prototyped and tested so far, a fourth is in production, and a fifth is in the late stages of design. Table 1 shows the critical features of these designs. Figure 3 shows representative results from a v3 module where the Tx eye is wide open at $10 \mathrm{~Gb} / \mathrm{s}$. The Rx sensitivity was also measured and found to easily meet the specification of $-13.1 \mathrm{dBm}$.

Table 1: Overview of in-house module designs. VCSELs and PD from two manufacturers (A and B) have been evaluated on these modules together with two optical coupling blocks (12-channel and $2 \mathrm{Tx}+2 \mathrm{Rx}$ ). Early prototypes had card-edge connectors while more recent designs use board-to-board (B2B) plug/socket connectors.

\begin{tabular}{|c|c|c|c|c|c|c|c|c|}
\hline \multirow[t]{2}{*}{ Version } & \multicolumn{2}{|c|}{$\mathrm{Tx}$} & \multicolumn{2}{|c|}{$\mathrm{Rx}$} & \multirow{2}{*}{$\begin{array}{c}\text { Coupling } \\
\text { block }\end{array}$} & \multirow{2}{*}{$\begin{array}{l}\text { Electrical } \\
\text { Connector }\end{array}$} & \multirow{2}{*}{$\begin{array}{l}\text { Target } \\
\text { height }\end{array}$} & \multirow[t]{2}{*}{ Status } \\
\hline & LDD & VCSEL & TIA & PD & & & & \\
\hline $\mathrm{v} 1$ & COTS & single (A) & GBTIA & single (A) & $12 \mathrm{ch}$. & SFP & - & Tested (9) \\
\hline v2 & COTS & single (A) & GBTIA & single (A) & $2+2 \mathrm{ch}$. & SFP & - & Tested (10) \\
\hline v3 & LDQ10 & array (A) & GBTIA & single (A) & $12 \mathrm{ch}$. & QSFP & - & Tested (7) \\
\hline $\mathrm{v} 3 \mathrm{~b}$ & LDQ10P & array $(\mathrm{A} / \mathrm{B})$ & GBTIA & single (A) & $12 \mathrm{ch}$. & QSFP & - & Tested (5/5) \\
\hline v4 & LDQ10 & array $(\mathrm{A} / \mathrm{B})$ & GBTIA & single (A) & $2+2 \mathrm{ch}$. & B2B & $2.5 \mathrm{~mm}$ & Prodn. (10) \\
\hline v5 & LDQ10 & array $(\mathrm{A} / \mathrm{B})$ & GBTIA & single (A) & $12 \mathrm{ch}$. & B2B & $4.0 \mathrm{~mm}$ & Design \\
\hline
\end{tabular}
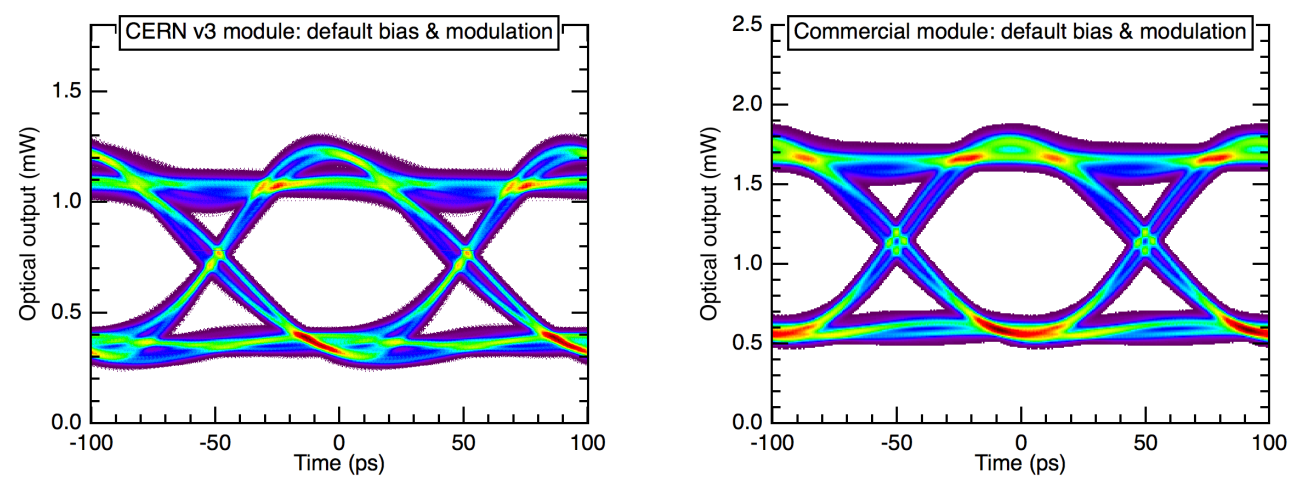

Figure 3: Respresentative results from prototype VTRx+ modules based on the LDQ10 laser driver operating at room temperature: (left) CERN v3 module; and (right) commercial module.

\subsection{COTS-based VTRx+module}

A Market Survey has been carried out within the CERN procurement framework in order to identify companies with the required know-how to produce high-speed optical transceiver modules that can be customised to meet the required dimensions of the VTRx+. The next step in the process is to solicit bids for the customisation of existing transceiver modules from the companies identified by the Market Survey. Once the bids have been received CERN will place development contracts with several manufacturers to supply VTRx+ prototypes within less than a year. 
During the Market Survey several companies have already included the CERN-supplied chipset of driver and TIA in their existing modules. This has been quite successful and a typical eye diagram from one channel of one device is shown in figure 3. Additional tests have been carried out showing that the devices operate within specifications over the entire required temperature range $\left(-35^{\circ} \mathrm{C}\right.$ to $\left.65^{\circ} \mathrm{C}\right)$. Test results from modules with two different VCSEL types have been obtained that show both can be driven successfully by the LDQ10 laser driver.

\section{Development schedule}

The VTRx+ module development is expected to be completed by then end of 2018 to allow 6-12 months of evaluation by potential users before the tendering process needs to begin in order to allow production to start in 2020. The development process will yield sufficient modules to allow reliability testing with the required sample sizes as well as the parts needed by the various user groups to validate their system designs.

\section{Summary and Conclusion}

The VL+ project has demonstrated the feasibility of operating optical link systems in the innermost regions of the HL-LHC experiments. Prototype VTRx+ front-end modules for this optical link have been shown to operate within the link specifications. Candidate components (VCSELs, laser driver, PINs, TIA) have also been evaluated for functional and environmental performance and found to operate within specifications. Details of the component tests (inc. radiation) will be subject to a future publication. The next step will be to show that modules meeting the final dimensional requirements can be prototyped and qualified, ready to go into production in early 2020.

\section{References}

[1] P. Moreira, The LpGBT project status and overview, in ACES 2016 - Fifth Common ATLAS CMS Electronics Workshop for LHC Upgrades, CERN, March, 2016.

[2] Z. Zeng, T. Zhang, G. Wang, P. Gui, S. Kulis, and P. Moreira, LDQ10: a compact ultra low-power radiation-hard $4 \times 10 \mathrm{~Gb} / \mathrm{s}$ driver array, Journal of Instrumentation 12 (2017), no. 02 P02020.

[3] M. Menouni, P. Gui, and P. Moreira, The GBTIA, a 5 Gbit/s Radiation-Hard Optical Receiver for the SLHC Upgrades, in Topical Workshop on Electronics for Particle Physics (TWEPP2009), 2009. Topical Workshop on Electronics for Particle Physics (TWEPP2009).

[4] L. Amaral, S. Dris, A. Gerardin, T. Huffman, C. Issever, A. J. Pacheco, M. Jones, S. Kwan, S. C. Lee, Z. Liang, T. Liu, Z. Meng, A. Prosser, S. Padadopoulos, I. Papakonstanstinou, C. Sigaud, S. Silva, C. Soos, P. Stejskal, J. Troska, F. Vasey, P. Vichoudis, T. Weidberg, A. Xiang, and J. Ye, The Versatile Link, a common project for super-LHC, Journal of Instrumentation 4 (2009), no. 12 P12003.

[5] F. Vasey, D. Hall, T. Huffman, S. Kwan, A. Prosser, C. Soos, J. Troska, T. Weidberg, A. Xiang, and J. Ye, The Versatile Link common project: feasibility report, Journal of Instrumentation 7 (2012), no. $01 \mathrm{C} 01075$. 